\title{
LHomme
}

L'HOMME Revue française d'anthropologie

$183 \mid 2007$

Comment être parents ?

\section{Agnès Fine \& Françoise-Romaine Ouellette, Le Nom dans les sociétés occidentales contemporaines}

Toulouse, Presses universitaires du Mirail, 2005, 252 p., bibl., ill., fig.

Valérie Feschet

\section{(2) OpenEdition}

\section{Journals}

Édition électronique

URL : http://journals.openedition.org/lhomme/9731

DOI : 10.4000//homme.9731

ISSN : 1953-8103

Éditeur

Éditions de l'EHESS

Édition imprimée

Date de publication : 1 septembre 2007

Pagination : 217-219

ISSN : 0439-4216

Référence électronique

Valérie Feschet, «Agnès Fine \& Françoise-Romaine Ouellette, Le Nom dans les sociétés occidentales contemporaines », L'Homme [En ligne], 183 | 2007, mis en ligne le 28 juin 2007, consulté le 24 septembre 2020. URL : http://journals.openedition.org//homme/9731 ; DOI : https://doi.org/10.4000/ Ihomme.9731

Ce document a été généré automatiquement le 24 septembre 2020.

(c) École des hautes études en sciences sociales 


\section{Agnès Fine \& Françoise-Romaine Ouellette, Le Nom dans les sociétés occidentales contemporaines}

Toulouse, Presses universitaires du Mirail, 2005, 252 p., bibl., ill., fig.

\section{Valérie Feschet}

1 CET OUVRAGE COLLECTIF, dirigé par Agnès Fine et Françoise-Romaine Ouellette, arrive fort à propos alors que la réforme de la transmission du nom de famille en France a été en application le $1^{\mathrm{er}}$ janvier 2005. Le livre rassemble dix contributions (parmi lesquelles il faut compter l'introduction pour la richesse de la synthèse qu'elle propose) sur les systèmes onomastiques contemporains. Tous les travaux, à l'exception de l'article d'Agnès Clerc-Renaud qui rend compte de l'importance des prénoms dans une localité du Nord Ceará (Brésil), portent sur la France et le Québec. La comparaison entre les deux est judicieuse car, au Québec, le Code civil permet de transmettre aux enfants, depuis 1980, le nom de l'un ou l'autre de ses parents ou les deux (sans précision quant à l'ordre retenu et les enfants d'une même famille peuvent recevoir chacun un nom différent).

2 On peut s'interroger sur la structuration de l'ouvrage qui ne met pas, à mon sens, suffisamment en valeur la richesse des données réunies mais il faut souligner qu'il s'agit là d'un ensemble de textes de très grande qualité, proposant des données de terrain précises et complémentaires qui, mises bout à bout, instruisent les mutations du système de nomination occidental. La première partie du livre rassemble des études sur la question de l'identification des personnes; la seconde insiste sur les interférences entre la nomination et l'affirmation des appartenances (à un sexe, à une communauté culturelle ou nationale, à une identité civile). À première vue, la partition de la problématique est classique (identifier des personnes d'une part et exprimer des existences spécifiques d'autre part) et le traitement des données reste dans le sillon des labours profonds qui ont pu être d'ores et déjà creusés sur le sujet. Toutefois, les apports de cet ouvrage sont conséquents. Ce recueil de textes rappelle que le nom personnel ne se résume pas seulement à un patronyme ou à un matronyme comme 
incitent à le penser les réformes actuelles, que le choix entre l'un ou l'autre du nom des parents pour nommer l'enfant n'est finalement pas grand-chose en regard de tout le reste et qu'il est nécessaire de prendre du recul vis-à-vis des différents processus idéologiques en œuvre...

Pour rentrer dans le vif du sujet, reprenons la dernière phrase du livre, concluant un texte signé par Sylvie Sagnes qui propose une analyse très éclairante sur la construction de la notion de " patrimoine onomastique ». L'auteur attire l'attention sur l'écart qu'il y a entre les arguments annoncés comme étant les moteurs de la réforme, de bon ton (l'égalité des sexes), et les motivations sous-jacentes, plus ou moins inconscientes, qui s'avèrent être d'une tout autre nature: "Car même si les modifications du droit en matière de nomination et d'état civil peuvent se donner les allures d'une adaptation aux évolutions de la famille et de la société, le progressisme dont les uns et les autres se drapent ne sert que d'alibi, voire de caution, à des élans plus secrets, à des crispations conservatrices et réactionnaires plus indicibles ». Sous des allures de modernité (l'égalité des sexes), les motivations de la réforme seraient donc aussi ancrées dans des logiques nationalistes, voire xénophobes, notre patronymie souffrant aux yeux de nombreuses associations et personnalités politiques, non seulement de la disparition des «noms rares » qui ne pouvaient plus être transmis mais aussi (et peut-être surtout) de l'intrusion et de la persistance d'occurrences nouvelles, débat passionnant dans lequel s'entrechoquent les Lebreton, Delattre, Martin, N'Guyen, Diawara, Mohamed... Cette analyse subtile des discours sur le « nom patrimoine » remet d'un seul coup tout à plat. Et si nous n'avions rien compris? Les réformateurs ne seraient-ils pas les plus "réactionnaires» et les plus "réactionnaires» les plus "progressistes»?

Quoi qu'il en soit, le prénom (qui fait l'objet de toutes les contributions de la première partie) semble rester un agent essentiel du processus d'identification. Au Brésil, par exemple, la partie du nom qui compte, aujourd'hui encore, celle qui vaut pour le tout, est le prénom qui structure jusqu'aux listes alphabétiques des annuaires (Agnès ClercRenaud)! En revanche, le prénom, qui figure sur les papiers d'identité des Manouches, est perçu par eux comme étant un "nom d'école ", c'est-à-dire celui qu'on utilise d'une manière générale dans les institutions mais sans plus. Le nom par lequel un Manouche est connu à l'intérieur de sa communauté est tout autre. Le « Romano Lap » est un nom d'intimité porteur de sens que seuls les proches peuvent saisir. Chaque nomination est une invention, jamais hasardeuse, qui s'appuie sur un jeu entre le son et le sens, à partir d'un matériel linguistique environnant. Ainsi "Tchounay» vient-il de «tonight », «Djina » est une variante du mot «Dji » qui signifie « le cœur » (Jean-Luc Poueyto)... Le prénom et/ou le "petit-nom » est essentiel, mais quand il n'est pas associé au nom, il peut être aussi le symptôme d'une défaillance filiale. Au Québec, les enfants nés dans le secret des origines et recueillis en institution n'avaient pas de noms de famille et ne portaient qu'un prénom (avant 1932), deux ensuite, l'un des prénoms valant pour «nom ». Ainsi, socialement marqués par leur «nom-prénom», les enfants illégitimes étaient facilement repérables et dramatiquement stigmatisés (Rose Dufour) ... Quant à la transmission traditionnelle des prénoms, déjà très étudiée, et qui n'obéit plus aux règles de naguère, on retiendra l'idée que si les patronymes sont un indice des appartenances nationales ou ethniques, il appartient néanmoins encore aux prénoms de spécifier les appartenances religieuses au sens large. En effet, malgré l'ouverture du "marché des prénoms", peu de Français inscrits dans une tradition familiale chrétienne puisent dans le registre des prénoms connotés comme musulmans et peu de 
musulmans de naissance choisissent pour leurs enfants des prénoms chrétiens (JeanPierre Albert).

5 Le prénom n'est pas seulement une manière d'identifier précisément les individus. Il permet également de construire les identités, en l'occurrence sexuelle. La deuxième partie de l'ouvrage commence par un texte de Louis Duchesne, démographe, qui rappelle que si le principal message d'un prénom est l'identification du sexe de la personne qui le porte, il existe néanmoins des prénoms mixtes (Frédéric/Frédérique, André/Andrée, Gabriel/Gabrielle...). On peut se demander si l'utilisation de ces prénoms non sexués est un indicateur social et culturel des frontières entre les sexes? La question est ambitieuse, l'énigme loin d'être résolue, mais la variation des usages, selon les lieux et les périodes (moins de $5 \%$ des garçons aujourd'hui en France reçoivent un prénom mixte contre près d'un quart à la fin des années 1930) mérite attention à l'heure où la constitution des sexes est au cœur des préoccupations sociales et scientifiques. Avec le travail de Denise Lemieux, il est cette fois question du « nom de famille » proprement dit attribué au premier enfant au Québec à la fin des années 1990. En 2002, soit plus de 20 ans après la réforme, $13 \%$ seulement des enfants se voyaient attribuer un nom composé ( $2 \%$ en 1982) et $1 \%$ des enfants reconnus par leur père avaient reçu le nom de leur mère. Le nom du père se maintient donc dans une écrasante majorité ce qui donne à réfléchir sur les motivations qui président au choix d'un autre type de nom. Josiane Le Gall et Deirdre Meintel offrent des éléments de réponse à partir d'une analyse des unions mixtes à Montréal où presque la moitié des enfants portent des noms composés pour répondre à leurs appartenances multiples... Une étude des pratiques contemporaines du nom marital, un usage perçu comme une obligation légale dans la société française, est ensuite proposée par Marie-Françoise Jeauffreau qui fait remarquer que le «nom marital » n'est pas l'équivalent du «nom conjugal » que l'on rencontre dans d'autres pays européens car il porte en lui une dissymétrie entre les sexes (problématique essentielle mais non traitée hélas !)...

Le « nom de famille » n'est donc que le fragment d'un système de nomination beaucoup plus vaste qu'il faut saisir dans son ensemble pour cerner avec justesse les tenants et les aboutissants des processus onomastiques contemporains. Je regrette que les «noms d'usage » que l'on porte juste le temps d'une vie mais que l'on ne transmet pas, les "surnoms ", les "petits noms ", les "pseudonymes", les «noms conjugaux " n'aient pas été abordés. Mais l'ensemble de ces travaux, sans nul doute, même si la problématique générale n'est pas conduite aussi fermement qu'on pourrait le souhaiter du début jusqu'à la fin de l'ouvrage, et s'il est parfois un peu difficile de ne pas perdre le fil des rapports entre «le nom, l'État, la famille et l'individu sexué », est une référence dorénavant incontournable pour conduire une réflexion, anthropologique ou civique, sur l'usage et la puissance du nom personnel dans les sociétés occidentales. 


\section{AUTEUR}

\section{VALÉRIE FESCHET}

Université Provence Aix-Marseille I, Institut d'ethnologie méditerranéenne et comparative, Aix-en-Provence.

Feschet@mmsh.univ-aix.fr 